\title{
Contribution au Recensement des Plantes Médicinales au Togo : Cas de la Région Maritime
}

\author{
Agody Mireille, \\ Bakoma Batomayena, \\ Batawila Komlan, \\ Wala Kpérkouma, \\ Dourma Marra, \\ Pereki Hodabalo, \\ Dimobe Kangbéni,
}

Faculté de Pharmacie, Université Cheik Anta Diop, Dakar

Faculté des Sciences de la Santé, Université de Lomé, Togo

Laboratoire de Botanique et Ecologie Végétale,

Faculté des Sciences, Université de Lomé, Togo

Bassene Emmanuel,

Faculté de Pharmacie, Université Cheik Anta Diop, Dakar

Akpagana Koffi,

Laboratoire de Botanique et Ecologie Végétale,

Faculté des Sciences, Université de Lomé, Togo

Doi:10.19044/esj.2019.v15n24p329 URL:http://dx.doi.org/10.19044/esj.2019.v15n24p329

\section{Resume}

L'enquête ethnobotanique menée sur neuf marchés de la région maritime du Togo a permis de recenser 106 espèces médicinales regroupées en 61 familles et 97 genres, dont 90 dicotylédones, 6 monocotylédones et une fougère. Ces différentes espèces interviennent dans le traitement de 63 pathologies, le paludisme et les troubles gynécologiques étant les plus fréquentes. Cette grande panoplie d'indications thérapeutiques montre le rôle important que les plantes jouent sur le plan médical. L'infusion et la décoction apparaissent comme les modes de préparation les plus utilisés par les tradithérapeutes. Plusieurs parties des plantes entrent dans ces différentes préparations. Les feuilles sont les plus utilisées. Ces résultats d'enquêtes ethnobotaniques pourront permettre une orientation éventuelle dans le choix des plantes cibles pour des pathologies données et la recherche de nouvelles molécules plus actives.

Mots-clés : Ethnobotanique, Pathologies, Région Maritime, Togo 


\title{
Contrabution to the Census of Medicinal Plants in Togo: Case of the Maritime Region
}

\author{
Agody Mireille, \\ Bakoma Batomayena, \\ Batawila Komlan, \\ Wala Kpérkouma, \\ Dourma Marra, \\ Pereki Hodabalo, \\ Dimobe Kangbéni,
}

Faculté de Pharmacie, Université Cheik Anta Diop, Dakar

Faculté des Sciences de la Santé, Université de Lomé, Togo

Laboratoire de Botanique et Ecologie Végétale,

Faculté des Sciences, Université de Lomé, Togo

Bassene Emmanuel,

Faculté de Pharmacie, Université Cheik Anta Diop, Dakar

Akpagana Koffi,

Laboratoire de Botanique et Ecologie Végétale,

Faculté des Sciences, Université de Lomé, Togo

\begin{abstract}
The ethnobotany survey conducted in nine markets of Togo maritime region leads to identification of 106 medicinal species in 61 families and 97 genera, including 90 dicotyledons, 6 monocotyledons, and one fern. These different species are used in the treatment of 63 pathologies, of which malaria and Gynecologic disorders are the most common. This vast array of therapeutic indications shows the important role that plants play in medical terms. The infusion and decoction appear as the most used methods of the traditional therapists. Several parts of plants fall into these different preparations. The leaves are the most used. These results of ethnobotanical survey will allow a possible orientation in the choice of target plants for given pathologies and the search for new molecules that are more active.
\end{abstract}

Keywords: Ethnobotany, Pathologies, Maritime region, Togo 


\section{Introduction}

La médecine dite traditionnelle est une méthode purement empirique de traitement des maladies. Elle se réduit le plus souvent à soupçonner les propriétés de guérison des affections humaines déterminées grâce à des substances généralement extraites de plantes. Ainsi, l'espoir en cette source végétale de remèdes ne cesse de motiver des investigations en vue de découvrir ou de préciser ses vertus thérapeutiques.

Or, l'étude des plantes médicinales s'avère une tâche ardue et urgente, en raison $\mathrm{du}$ contexte sociologique de leur utilisation et du vaste champ d'investigation qu'elles offrent. De plus, la transmission de cet ensemble de données repose sur des générations de guérisseurs et guérisseuses expérimentés, qui se font de plus en plus rares et dont le savoir se dégrade, les rendant de moins en moins dévoués à la cause de la santé, les rentrées d'argent étant aujourd'hui leur préoccupation majeure.

Il n'empêche que le traitement des maladies par les plantes demeure le moyen le plus usité dans nos pays africains à forte population rurale. Au Togo principalement, il est à noter que 60 à $80 \%$ de la population utilise les plantes médicinales pour se soigner (Batawila, 2005 ; Sari , 2006) ; ce qui démontre la place importante qu'occupent les herboristes dans la couverture sanitaire du pays.

Le souci, en choisissant comme sujet d'étude le recensement des plantes médicinales vendues dans la Région Maritime du Togo, est d'apporter une modeste contribution à un recensement objectif des espèces vendues sur les marchés de ladite région, à majorité rurale (79\%). C'est d'ailleurs ce qui explique (en partie) que, malgré la profusion des cabinets médicaux et des pharmacies dans Lomé et ses environs, la sécurité sanitaire n'est pas garantie pour certaines couches de la population. En outre, le manque de ressources financières et la situation actuelle de crise aiguë n'améliore pas cet état de fait. On assiste, de plus en plus, à un repli de cette population (même urbaine) vers les charlatans, les féticheurs et surtout les herboristes.

D'après le Dictionnaire Larousse 2005, les herboristes sont des « personnes non diplômées en pharmacie qui vendent des plantes médicinales au public ». Ces personnes au Togo, et principalement à Lomé, sont surtout des femmes qui vendent sur les marchés locaux des plantes exposées sur des étalages dans un fouillis extraordinaire qu'elles parviennent cependant à gérer.

L'extravagance des étalages et la quantité de spécimens de plantes qu'on y compte suscitent un vif intérêt et une curiosité toute scientifique qui amènent à se poser moult questions : Ne serait-il pas avantageux de répertorier l'ensemble des plantes que l'on trouve dans les marchés, chez les herboristes ? Existe-t-il vraiment un traitement à tous les maux, sur ces étalages «vertigineux »? Les herboristes locaux maîtrisent-ils vraiment les modes d'emploi de toutes ces plantes ? Les populations ressentent-elles vraiment de 
la satisfaction en recourant aux herboristes ? Autant de questionnements et tout autant de préoccupations dont peut procéder une étude orientée vers des objectifs principal et spécifiques.

L'objectif principal est de recenser systématiquement les plantes utilisées dans le traitement des maladies les plus courantes dans la région maritime au Togo. Il s'agit entre autres de dresser une liste plus ou moins exhaustive des plantes médicinales, que l'on rencontre sur les marchés de la Région Maritime, et de leurs indications thérapeutiques.

\section{Méthodologie}

\section{Choix des marchés}

Limitée au départ au seul cadre de Lomé pour établir un inventaire détaillé des plantes vendues sur les marchés, cette étude s'est étendue à d'autres villes de la région maritime. Ces villes sont connues pour leur richesse en plantes médicinales et donc pourvoyeuses de la capitale. Il s'agit des marchés de Tsévié (à 35km de Lomé), Noépé (30km), Tabligbo $(77 \mathrm{~km})$, et Vogan $(50 \mathrm{~km})$.

Cependant, Lomé étant une agglomération plus importante, cinq marchés y ont été sélectionnés : le marché de Bè, le marché d'Attikpodji, le marché d'Akodesséwa, le marché d'Adidogomé et le marché d'Hanoukopé. Ces marchés ont été choisis du fait de l'importance de leurs rayons «Plantes Médicinales ». Les autres villes échantillons ont été visitées le jour de la semaine qui correspond à leur jour de marché.

\section{Étude qualitative}

Un inventaire des drogues végétales observées sur les étalages a été effectué. Pour ce faire, des fiches préétablies ont été renseignées sur les plantes médicinales, les vendeurs et les acheteurs de ces plantes. Au retour du terrain, ces fiches ont été dépouillées et les informations recueillies ont été classées suivant les objectifs spécifiques de l'étude.

Les plantes entières et les parties de plantes (feuilles, tiges, racines, écorces de tronc ou de racine, graine, fleurs) ont été identifiées sur place à l'aide d'un Botaniste. Celles qui n'ont pu être déterminées ont été achetées puis déterminées à l'herbarium du Laboratoire de Botanique et Écologie Végétale de l'Université de Lomé où des échantillons d'herbier ont été déposés.

La nomenclature suivie est celle de Hutchinson et Dalziel (1954 1972) et Brunel et al. (1984). Les renseignements sur les plantes ont été complétés généralement par le nom scientifique de la plante.

Chaque tableau ou fiche contient les informations suivantes :

- La photo (si possible) : certaines espèces n’ont pas pu être photographiées pour des raisons matérielles. 
- Le nom vernaculaire de la plante, souvent en Ewé ou en Mina, les deux dialectes les plus parlés dans la région maritime du Togo, cadre de l'étude. Cependant, certains de ces noms sont en Fon (la langue la plus parlée dans le Sud du Bénin), ou en Yoruba (langue des Nagos originaires des régions frontières du Bénin avec le Nigeria).

- Les indications pour lesquelles la plante est utilisée.

- Le mode d'emploi, le mode de préparation et la posologie groupés tous ensemble. Il s'agit, ici, de l'utilisation indigène et traditionnelle faite de la plante. Ce sont des connaissances plus ou moins empiriques, soumises ou non à des recherches scientifiques. De plus, ces informations ont été recueillies auprès des femmes des marchés ou des tradithérapeutes qui ne sont vraiment pas disposés à révéler tous leurs secrets.

-Les particularités de la plante : il s’agit généralement des «vertus » de la plante qualifiées de «médico - magiques ». Il a été jugé intéressant de mentionner les caractéristiques de certaines de ces plantes que les tradithérapeutes ont bien voulu révéler. Pour d'autres, ils ont obstinément caché ces vertus.

\section{Resultats}

\section{Diversité des plantes médicinales de la région maritime}

Les enquêtes ethnobotaniques sur les marchés (cinq à Lomé et quatre dans les villes échantillons de la région maritime) ont permis de recenser 106 espèces. Ces dernières sont regroupées en 61 familles et 97 genres.

Les familles les plus représentées sont les Caesalpiniaceae (six espèces), les Euphorbiaceae (six espèces), les Amaranthaceae (cinq espèces), les Apocynaceae (cinq espèces), et les Rubiaceae (quatre espèces).

Pour les autres familles six sont représentées par trois espèces. Les familles représentées par deux espèces sont au nombre de treize et celles représentées par une espèce sont au nombre de 37.

\section{Indications thérapeutiques}

Les cent - six (106) espèces recensées sont indiquées dans le traitement de 63 différentes pathologies. Dix pathologies sont fréquemment observées avec en tête le paludisme. Ensuite viennent dans l'ordre : les troubles gynécologiques, le rhumatisme, les dermatoses, le diabète, les parasitoses intestinales, les troubles hépatobiliaires, l'anémie, les MST (maladies sexuellement transmissibles), les troubles hépatiques.

Le paludisme, les dermatoses, les parasitoses intestinales, les MST sont des pathologies à fort impact dans les régions tropicales, ce qui justifierait que ces pathologies soient fréquemment observées. Selon les observations faites sur le terrain, les rhumatismes ont également une forte fréquence qui 
s'explique par la part importante des personnes âgées parmi les clients et les patients des vendeuses de plantes médicinales et des tradithérapeutes.

Les affections les moins rencontrées sur le terrain sont au nombre de 20. Parmi ces affections on peut citer les cas de brûlure au $3^{\text {ème }}$ degré, d'anorexie, de choléra, de cancer du poumon, de cancer du foie. La faible fréquence de ces affections s'explique par le fait que celles-ci soient souvent rares comme les cancers, ou suivies de près par les autorités de la santé comme indiqué sur la Figure 1.

Il y a pas mal de plantes qui ont un pouvoir médico-magique. Selon la répartition des espèces médicinales suivant les différentes pathologies traitées, certaines plantes sont impliquées dans le traitement de plusieurs maladies. C'est le cas par exemple de Schwenkia americana et d'Annona senegalensis citées pour le traitement de dix et huit pathologies respectivement. Carica papaya, Jatropha gossypifolia, Ocimum canum sont indiquées chacune dans le traitement de sept maladies. Les autres espèces recensées interviennent d'une manière générale dans le traitement d'au moins trois ou quatre pathologies (Figure 2).

Pour permettre de retrouver aisément les espèces utilisées dans le traitement d'une pathologie donnée, la répartition des espèces suivant les différentes pathologies est consignée dans le tableau 1 (et ce pour dix des pathologies les plus courantes).

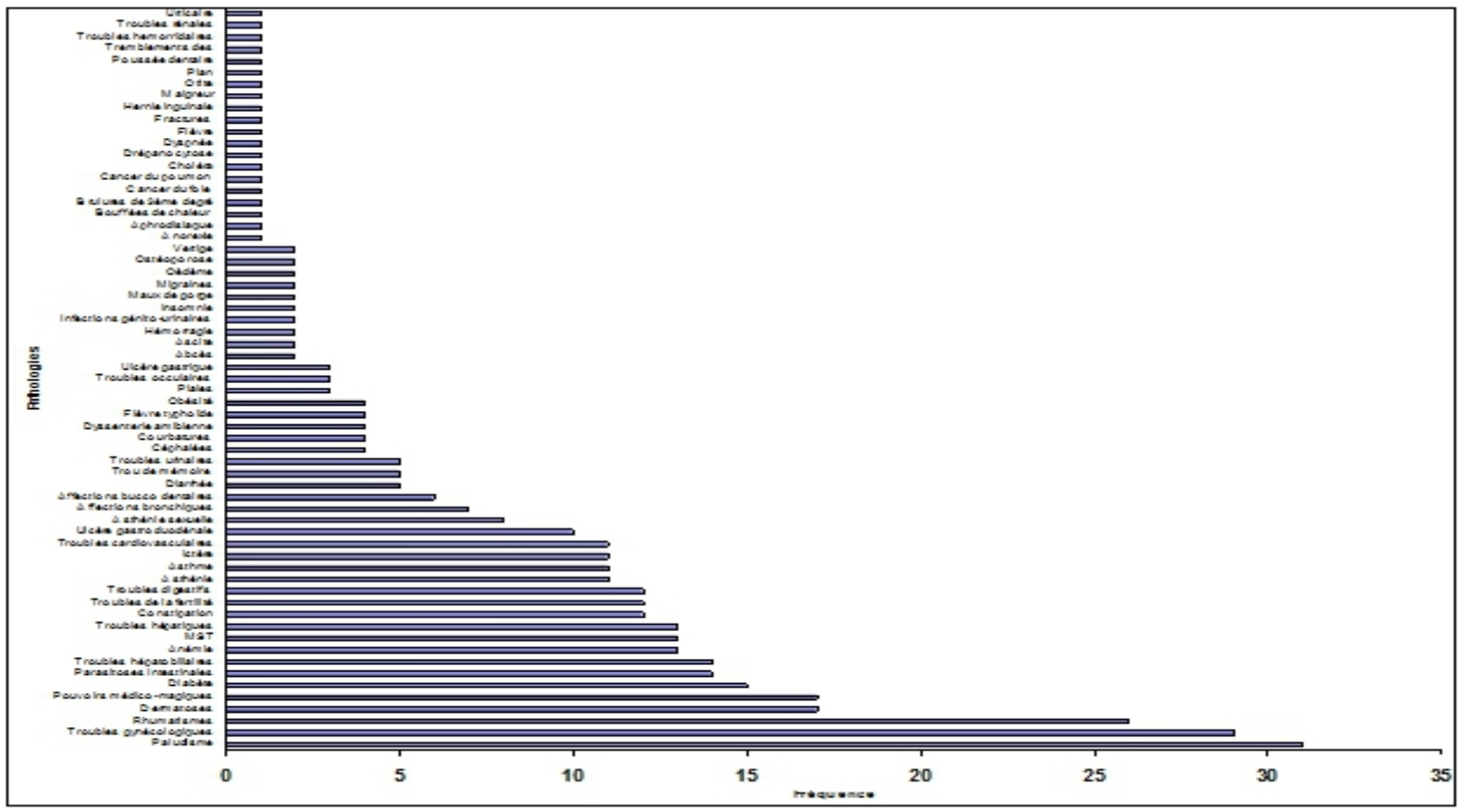

Figure 1. Fréquence des différentes pathologies traitées. 


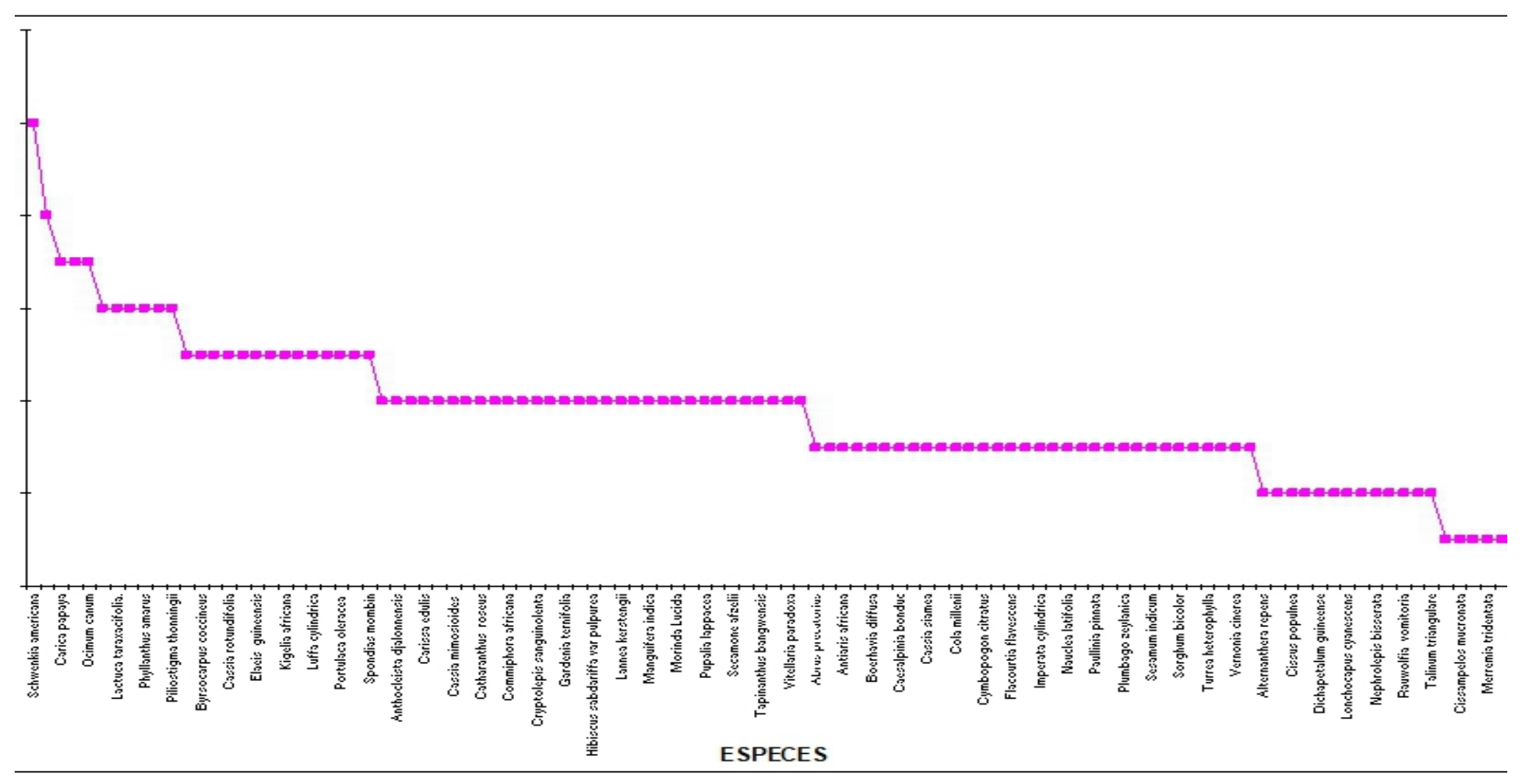

Figure 2. Distribution des espèces suivant les pathologies traitées.

\begin{tabular}{|c|c|c|c|c|}
\hline \multicolumn{5}{|c|}{ Abréviations : } \\
\hline Fr : Fruits & $\begin{array}{r}\mathrm{T}: \text { Tronc G : Graines } \\
\text { Ti : Tige }\end{array}$ & $\begin{array}{l}\mathrm{P} \overline{\mathrm{E}: \text { Plante Entière }} \\
\mathrm{F}: \text { Feuilles }\end{array}$ & $\begin{array}{l}\text { PA : Partie aérienne } \\
\text { AL : Application locale }\end{array}$ & E : Écorces \\
\hline
\end{tabular}


Tableau 1. Répartition des espèces par pathologie (cas de dix pathologies)

\begin{tabular}{|c|c|c|c|c|c|}
\hline $\begin{array}{l}\text { PATHOLOG } \\
\text { IE }\end{array}$ & NOM SCIENTIFIQUE & FAMILLE & NOM VERNACULAIRE & $\begin{array}{l}\text { PARTIE } \\
\text { UTILE }\end{array}$ & MODE \\
\hline $\begin{array}{l}\text { D } \\
\text { c } \\
\text { 믕 } \\
\text { 咅 }\end{array}$ & $\begin{array}{l}\text { Abrus precatorius } \\
\text { Adansonia digitata } \\
\text { Annona senegalensis } \\
\text { Carica papaya } \\
\text { Cassia occidentalis } \\
\text { Cassia rotundifolia } \\
\text { Cassia siamea } \\
\text { Cola millenii } \\
\text { Croton zambesicus } \\
\text { Cryptolepis sanguinolenta } \\
\text { Cymbopogon citratus } \\
\text { Dialium guineense } \\
\text { Dichapetalum guineense } \\
\text { Dyschoriste perrottetii } \\
\text { Elaeis guineensis } \\
\text { Gomphrena celosioides } \\
\text { Hibiscus sabdariffa } \\
\text { Jatropha gossypifolia } \\
\text { Lippia multiflora } \\
\text { Luffa cylindrica } \\
\text { Mondia whitei } \\
\text { Morinda Lucida } \\
\text { Nauclea latifolia } \\
\text { Newbouldia laevis } \\
\text { Parquetina nigrescens } \\
\text { Piliostigma thonningii } \\
\text { Psidium guineense } \\
\text { Schwenkia americana } \\
\text { Spondias mombin } \\
\text { Uvaria chamae } \\
\text { Vernonia cinerea }\end{array}$ & $\begin{array}{l}\text { Papilionaceae } \\
\text { Bombacaceae } \\
\text { Annonaceae } \\
\text { Caricaceae } \\
\text { Ceaesalpiniaceae } \\
\text { Ceaesalpiniaceae } \\
\text { Ceaesalpiniaceae } \\
\text { Sterculiaceae } \\
\text { Euphorbiaceae } \\
\text { Périplocaceae } \\
\text { Poaceae } \\
\text { Ceaesalpiniaceae } \\
\text { Chailletiaceae } \\
\text { Acanthaceae } \\
\text { Arecaceae } \\
\text { Amaranthaceae } \\
\text { Malvaceae } \\
\text { Euphorbiaceae } \\
\text { Verbaceae } \\
\text { Cucurbitaceae } \\
\text { Menispermaceae } \\
\text { Rubiaceae } \\
\text { Rubiaceae } \\
\text { Bignoniaceae } \\
\text { Periplocaceae } \\
\text { Ceaesalpiniaceae } \\
\text { Myrtaceae } \\
\text { Solanaceae } \\
\text { Anacardiaceae } \\
\text { Annonaceae } \\
\text { Asteraceae }\end{array}$ & $\begin{array}{l}\text { Dzedzekoudzen } \\
\text { Adido tsi } \\
\text { Anyigli tsi } \\
\text { Adouba tsi } \\
\text { Bessissan } \\
\text { Azin gbé } \\
\text { Sanguera tsi } \\
\text { kpando } \\
\text { Gbléli } \\
\text { Eke dzen } \\
\text { Tigbé } \\
\text { Ati toe tsi } \\
\text { Ati hali } \\
\text { Aditi } \\
\text { Dé tsi } \\
\text { Papa Tahé } \\
\text { Gnanto dzen } \\
\text { Baba ti djin } \\
\text { Avloti } \\
\text { Awonli koussa } \\
\text { Kanabo } \\
\text { Zanclam } \\
\text { Nyimo } \\
\text { Kpatima } \\
\text { Atonboka } \\
\text { Eklon } \\
\text { Gbèbè tsi } \\
\text { Tronsi poha } \\
\text { Aklikon tsi } \\
\text { Agba nan tsi } \\
\text { Hounsikonou }\end{array}$ & $\begin{array}{l}\text { PA } \\
\text { Fr } \\
R \\
F \\
P E \\
P A \\
F \\
F \\
F \\
R \\
P A \\
F+F r \\
F \\
P A \\
R \\
P E \\
P A \\
F \\
P A \\
F \\
R \\
F \\
P A \\
F \\
F ; F \\
F \\
P A \\
P E \\
F \\
E+F \\
P A\end{array}$ & $\begin{array}{l}\text { Infusion } \\
\text { Macération } \\
\text { Décoction } \\
\text { Infusion } \\
\text { Décoction } \\
\text { Infusion } \\
\text { Infusion } \\
\text { Décoction sommaire } \\
\text { Décoction } \\
\text { Décoction sommaire } \\
\text { Infusion } \\
\text { Infusion } \\
\text { Infusion } \\
\text { Infusion } \\
\text { Décoction } \\
\text { Décoction sommaire } \\
\text { Décoction sommaire } \\
\text { Infusion } \\
\text { Infusion } \\
\text { Infusion } \\
\text { Décoction } \\
\text { Infusion } \\
\text { Décoction sommaire } \\
\text { Macération } \\
\text { Infusion; macération } \\
\text { Infusion } \\
\text { Infusion } \\
\text { Infusion } \\
\text { Macération } \\
\text { Décoction } \\
\text { Infusion }\end{array}$ \\
\hline
\end{tabular}




\begin{tabular}{|c|c|c|c|c|c|}
\hline 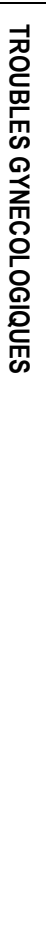 & $\begin{array}{l}\text { Annona senegalensis } \\
\text { Anthocleista djalonnensis } \\
\text { Antiaris africana } \\
\text { Carica papaya } \\
\text { Cassia mimosioides } \\
\text { Chassalia kolly } \\
\text { Cissampelos mucronata } \\
\text { Dyschoriste perrottetii } \\
\text { Euphorbia hirta } \\
\text { Fagara zanthoxyloides } \\
\text { Ficus exasperata } \\
\text { Flacourtia flavescens } \\
\text { Kigelia africana } \\
\text { Lannea kerstengii } \\
\text { Luffa cylindrica } \\
\text { Morinda Lucida } \\
\text { Newbouldia laevis } \\
\text { Parkia biglobosa } \\
\text { Phyllanthus amarus } \\
\text { Phyllanthus niruri } \\
\text { Piliostigma thonningii } \\
\text { Pteleopsis suberosa } \\
\text { Sansevieria liberica } \\
\text { Secamone afzelii } \\
\text { Spondias mombin } \\
\text { Tapinanthus bangwensis } \\
\text { Triclisia subcordata } \\
\text { Vernonia cinerea } \\
\text { Vitellaria paradoxa }\end{array}$ & $\begin{array}{l}\text { Annonaceae } \\
\text { Loganiaceae } \\
\text { Moraceae } \\
\text { Caricaceae } \\
\text { Ceaesalpiniaceae } \\
\text { Rubiaceae } \\
\text { Menispermaceae } \\
\text { Acanthaceae } \\
\text { Euphorbiaceae } \\
\text { Rutaceae } \\
\text { Moraceae } \\
\text { Flacourtiaceae } \\
\text { Bignoniaceae } \\
\text { Anacardiaceae } \\
\text { Cucurbitaceae } \\
\text { Rubiaceae } \\
\text { Bignoniaceae } \\
\text { Mimosaceae } \\
\text { Euphorbiaceae } \\
\text { Euphorbiaceae } \\
\text { Ceaesalpiniaceae } \\
\text { Combretaceae } \\
\text { Agavaceae } \\
\text { Asclepiadaceae } \\
\text { Anacardiaceae } \\
\text { Loranthaceae } \\
\text { Menispermaceae } \\
\text { Asteraceae } \\
\text { Sapotaceae }\end{array}$ & $\begin{array}{l}\text { Anyigli tsi } \\
\text { Gbolo ba } \\
\text { Logotsi } \\
\text { Adouba tsi } \\
\text { Kilè fimitsè } \\
\text { Ation do koé } \\
\text { Djokodjè } \\
\text { Aditi } \\
\text { Anon si gbé } \\
\text { Hè tsi } \\
\text { Sasaplala } \\
\text { Hlohoui } \\
\text { Gna kpé kpé } \\
\text { Melonkou } \\
\text { Awonli koussa } \\
\text { Zanclam } \\
\text { Kpatima } \\
\text { Ewati } \\
\text { Ehlin } \\
\text { Hlenwe } \\
\text { Eklon } \\
\text { Sisinon } \\
\text { Yodo bo tsi } \\
\text { Anon tsi ko } \\
\text { Aklikon tsi } \\
\text { Lolo gblé ati } \\
\text { katcha soè } \\
\text { Hounsikonou } \\
\text { Yokou tsi }\end{array}$ & $\begin{array}{l}R \\
T+R \\
T+R \\
F r \\
P A \\
R \\
F \\
P A \\
P E \\
R \\
R \\
R \\
F r+T ; T \\
T \\
F \\
R+T+F \\
T+R \\
F \\
P A \\
P A \\
F+G+R \\
T \\
R ; F \\
P A \\
T \\
P A \\
R \\
P A \\
T ; T\end{array}$ & $\begin{array}{l}\text { Décoction } \\
\text { Décoction } \\
\text { Décoction } \\
\text { Macération } \\
\text { Infusion } \\
\text { Poudre } \\
\text { Infusion } \\
\text { Infusion } \\
\text { Infusion } \\
\text { Décoction } \\
\text { Décoction } \\
\text { Décoction } \\
\text { Décoction } \\
\text { Décoction } \\
\text { Macération } \\
\text { Décoction } \\
\text { Décoction } \\
\text { Décoction } \\
\text { Infusion } \\
\text { Infusion } \\
\text { Décoction } \\
\text { Décoction } \\
\text { Décoction; macération } \\
\text { Décoction } \\
\text { Décoction } \\
\text { Décoction } \\
\text { Alcoolature } \\
\text { Infusion } \\
\text { Décoction ; Infusion }\end{array}$ \\
\hline
\end{tabular}




\begin{tabular}{|c|c|c|c|c|c|}
\hline 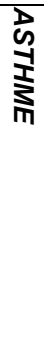 & $\begin{array}{l}\text { Adansonia digitata } \\
\text { Dialium guineense } \\
\text { Euphorbia hirta } \\
\text { Kigelia africana } \\
\text { Leptadenia hastata } \\
\text { Mangifera indica } \\
\text { Morinda Lucida } \\
\text { Paullinia pinnata } \\
\text { Pupalia lappacea } \\
\text { Sesamum indicum } \\
\text { Tapinanthus bangwensis }\end{array}$ & $\begin{array}{l}\text { Bombacaceae } \\
\text { Ceaesalpiniaceae } \\
\text { Euphorbiaceae } \\
\text { Bignoniaceae } \\
\text { Asclepiadaceae } \\
\text { Anacardiaceae } \\
\text { Rubiaceae } \\
\text { Sapindaceae } \\
\text { Amaranthaceae } \\
\text { Pedaliaceae } \\
\text { Loranthaceae }\end{array}$ & $\begin{array}{l}\text { Adido tsi } \\
\text { Ati toe tsi } \\
\text { Anon si gbé } \\
\text { Gna kpé kpé } \\
\text { Mitigbe } \\
\text { Mangotsi } \\
\text { Zanclam } \\
\text { Okuika } \\
\text { Miti miti } \\
\text { Foudrin foudrin } \\
\text { Lolo gblé ati }\end{array}$ & $\begin{array}{l}\mathrm{F} \\
\mathrm{R}+\mathrm{T}+\mathrm{F} \\
\mathrm{PE} \\
\mathrm{T} \\
\mathrm{PE} \\
\mathrm{F} \\
\mathrm{T} \\
\mathrm{R} \\
\mathrm{PA} \\
\mathrm{F} \\
\mathrm{PA}\end{array}$ & $\begin{array}{l}\text { Infusion } \\
\text { Décoction sommaire } \\
\text { Infusion } \\
\text { Infusion } \\
\text { Infusion } \\
\text { Ingestion } \\
\text { Décoction } \\
\text { Décoction } \\
\text { Infusion } \\
\text { Infusion } \\
\text { Décoction }\end{array}$ \\
\hline 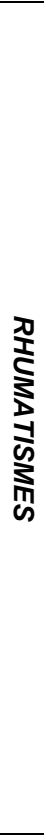 & $\begin{array}{c}\text { Annona senegalensis } \\
\text { Borassus aethiopum } \\
\text { Cardiospermum halicacabum } \\
\text { Carissa edulis } \\
\text { Cassia occidentalis } \\
\text { Clausena anisata } \\
\text { Cocos nucifera } \\
\text { Cola millenii } \\
\text { Cryptolepis sanguinolenta } \\
\text { Dialium guineense } \\
\text { Dichapetalum guineense } \\
\text { Euphorbia hirta } \\
\text { Fagara zanthoxyloides } \\
\text { Gardenia ternifolia } \\
\text { Gomphrena celosioides } \\
\text { Nephrolepis biserrata } \\
\text { Ocimum canum } \\
\text { Ocimum gratissimum } \\
\text { Parquetina nigrescens } \\
\text { Schwenkia americana } \\
\text { Scoparia dulcis } \\
\text { Secamone afzelii } \\
\text { Sida linifolia } \\
\text { Spathodea campanulata } \\
\text { Vitellaria paradoxa } \\
\text { Waltheria Indica }\end{array}$ & $\begin{array}{c}\text { Annonaceae } \\
\text { Arecaceae } \\
\text { Sapindaceae } \\
\text { Apocynaceae } \\
\text { Ceaesalpiniaceae } \\
\text { Rutaceae } \\
\text { Arecaceae } \\
\text { Sterculiaceae } \\
\text { Periplocaceae } \\
\text { Ceaesalpiniaceae } \\
\text { Chailletiaceae } \\
\text { Euphorbiaceae } \\
\text { Rutaceae } \\
\text { Rubiaceae } \\
\text { Amaranthaceae } \\
\text { Fougères } \\
\text { Labieae } \\
\text { Labieae } \\
\text { Periplocaceae } \\
\text { Solanaceae } \\
\text { Scrophulariaceae } \\
\text { Asclepiadaceae } \\
\text { Malvaceae } \\
\text { Bignoniaceae } \\
\text { Sapotaceae } \\
\text { Sterculiaceae } \\
\end{array}$ & $\begin{array}{l}\text { Anyigli tsi } \\
\text { Ago tsi } \\
\text { Otoka } \\
\text { Boetcho } \\
\text { Bessissan } \\
\text { Ida } \\
\text { Netsi=enetsi } \\
\text { kpando } \\
\text { Eke dzen } \\
\text { Ati toe tsi } \\
\text { Ati hali } \\
\text { Anon si gbé } \\
\text { Hè tsi } \\
\text { Fliflé tsi } \\
\text { Papa Tahé } \\
\text { edéhonlowè } \\
\text { Ahame } \\
\text { Essroun } \\
\text { Atonboka } \\
\text { Tronsi poha } \\
\text { Vivima } \\
\text { Anon tsi ko } \\
\text { Odoé ogbigbo wo } \\
\text { Adassi golo } \\
\text { Yokou tsi } \\
\text { Adouwè tsi }\end{array}$ & $\begin{array}{c}R \\
T \\
\text { Lianes } \\
P A+R \\
P E \\
F \\
F \\
F \\
R \\
R+T+F \\
F \\
P E \\
R \\
T i+R \\
P E \\
P A \\
P A \\
F I \\
R \\
P E \\
P A \\
P A \\
P E \\
T \\
T \\
F+R\end{array}$ & $\begin{array}{l}\text { Décoction } \\
\text { Décoction } \\
\text { Infusion } \\
\text { Décoction } \\
\text { Décoction } \\
\text { Infusion } \\
\text { Décoction sommaire } \\
\text { Décoction sommaire } \\
\text { Décoction sommaire } \\
\text { Décoction sommaire } \\
\text { Infusion } \\
\text { Infusion } \\
\text { Décoction } \\
\text { Décoction } \\
\text { Décoction sommaire } \\
\text { Infusion } \\
\text { Infusion } \\
\text { Infusion } \\
\text { A L } \\
\text { Infusion } \\
\text { Infusion } \\
\text { Infusion } \\
\text { Infusion } \\
\text { Décoction } \\
\text { Décoction } \\
\text { Décoction }\end{array}$ \\
\hline
\end{tabular}




\begin{tabular}{|c|c|c|c|c|c|}
\hline $\begin{array}{l}\frac{0}{8} \\
\frac{\mathbf{m}}{\mathbf{m}} \\
\frac{\mathbf{m}}{\operatorname{m}}\end{array}$ & $\begin{array}{c}\text { Aerva lanata } \\
\text { Bambusa vulgaris } \\
\text { Catharanthus roseus } \\
\text { Chassalia kolly } \\
\text { Cola millenii } \\
\text { Cryptolepis sanguinolenta } \\
\text { Gardenia ternifolia } \\
\text { Imperata cylindrica } \\
\text { Lactuca taraxacifolia. } \\
\text { Momordica charantia } \\
\text { Phyllanthus amarus } \\
\text { Phyllanthus niruri } \\
\text { Pleiocarpa pycnantha } \\
\text { Scoparia dulcis } \\
\text { Vernonia amygdalina }\end{array}$ & $\begin{array}{l}\text { Amaranthaceae } \\
\text { Poaceae } \\
\text { Apocynaceae } \\
\text { Rubiaceae } \\
\text { Sterculiaceae } \\
\text { Periplocaceae } \\
\text { Rubiaceae } \\
\text { Gramineae } \\
\text { Compositeae } \\
\text { Cucurbitaceae } \\
\text { Euphorbiaceae } \\
\text { Euphorbiaceae } \\
\text { Apocynaceae } \\
\text { Scrophulariaceae } \\
\text { Compositeae }\end{array}$ & $\begin{array}{l}\text { Akoèma } \\
\text { Plampo tsi } \\
\text { Netimè flower vi } \\
\text { Ation do koé } \\
\text { kpando } \\
\text { Eke dzen } \\
\text { Fliflé tsi } \\
\text { Ebe dzin } \\
\text { Awonto } \\
\text { Agnagna } \\
\text { Ehlin } \\
\text { Hlenwe } \\
\text { Avlokui gbe } \\
\text { Vivima } \\
\text { Aloma }\end{array}$ & $\begin{array}{c}\text { PE } \\
F \\
R \\
P A \\
R \\
R \\
F \\
R \\
F \\
P A \\
P A \\
P A \\
T+F \\
P A \\
P A\end{array}$ & $\begin{array}{c}\text { Infusion } \\
\text { Infusion } \\
\text { Infusion } \\
\text { Infusion } \\
\text { Décoction } \\
\text { Décoction sommaire } \\
\text { Infusion } \\
\text { Infusion } \\
\text { Infusion } \\
\text { Infusion } \\
\text { Infusion } \\
\text { Infusion } \\
\text { Infusion } \\
\text { Infusion } \\
\text { Infusion }\end{array}$ \\
\hline 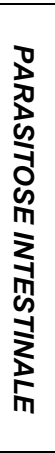 & $\begin{array}{c}\text { Alternanthera repens } \\
\text { Annona senegalensis } \\
\text { Carica papaya } \\
\text { Euphorbia hirta } \\
\text { Holarrhena floribunda } \\
\text { Indigofera astragalina } \\
\text { Leptadenia hastata } \\
\text { Momordica charantia } \\
\text { Phyllanthus amarus } \\
\text { Phyllanthus niruri } \\
\text { Pleiocarpa pycnantha } \\
\text { Portulaca oleracea } \\
\text { Schwenkia americana } \\
\text { Uvaria chamae }\end{array}$ & $\begin{array}{l}\text { Amaranthaceae } \\
\text { Annonaceae } \\
\text { Caricaceae } \\
\text { Euphorbiaceae } \\
\text { Apocynaceae } \\
\text { Papillionnaceae } \\
\text { Asclepiadaceae } \\
\text { Cucurbitaceae } \\
\text { Euphorbiaceae } \\
\text { Euphorbiaceae } \\
\text { Apocynaceae } \\
\text { Portulacaceae } \\
\text { Solanaceae } \\
\text { Annonaceae }\end{array}$ & $\begin{array}{c}\text { Abaklin } \\
\text { Anyigli tsi } \\
\text { Adouba tsi } \\
\text { Anon si gbé } \\
\text { Sé Sé hou } \\
\text { Avlo kui gbé } \\
\text { Mitigbe } \\
\text { Agnagna } \\
\text { Ehlin } \\
\text { Hlenwe } \\
\text { Avlokui gbe } \\
\text { Aflavi } \\
\text { Tronsi poha } \\
\text { Agba nan tsi }\end{array}$ & $\begin{array}{c}\text { PA } \\
R \\
G \\
P E \\
T \\
P A \\
P E \\
P A \\
P A \\
P A \\
T+F \\
P A \\
P E \\
\text { Ec + F }\end{array}$ & $\begin{array}{l}\text { Infusion } \\
\text { Décoction } \\
\text { Macération } \\
\text { Infusion } \\
\text { Décoction } \\
\text { Infusion } \\
\text { Infusion } \\
\text { Infusion } \\
\text { Infusion } \\
\text { Infusion } \\
\text { Infusion } \\
\text { Ingestion } \\
\text { Infusion } \\
\text { Décoction }\end{array}$ \\
\hline
\end{tabular}




\begin{tabular}{|c|c|c|c|c|c|}
\hline 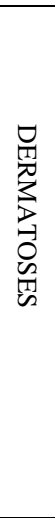 & $\begin{array}{c}\text { Abrus precatorius } \\
\text { Aloe buettneri } \\
\text { Alternanthera sessilis } \\
\text { Cassia alata } \\
\text { Cassia occidentalis } \\
\text { Citrus aurantifolia } \\
\text { Commiphora africana } \\
\text { Croton zambesicus } \\
\text { Ficus exasperata } \\
\text { Heliotropium indicum } \\
\text { Jatropha gossypifolia } \\
\text { Momordica charantia } \\
\text { Plumbago zeylanica } \\
\text { Pteleopsis suberosa } \\
\text { Schwenkia americana } \\
\text { Vitex doniana }\end{array}$ & $\begin{array}{c}\text { Papilionaceae } \\
\text { Liliaceae } \\
\text { Amaranthaceae } \\
\text { Ceaesalpiniaceae } \\
\text { Ceaesalpiniaceae } \\
\text { Rutaceae } \\
\text { Burseraceae } \\
\text { Euphorbiaceae } \\
\text { Moraceae } \\
\text { Borraginaceae } \\
\text { Euphorbiaceae } \\
\text { Cucurbitaceae } \\
\text { Plumbaginaceae } \\
\text { Combretaceae } \\
\text { Solanaceae } \\
\text { Verbenaceae }\end{array}$ & $\begin{array}{l}\text { Dzedzekoudzen } \\
\text { adi adi } \\
\text { Tosroingbé } \\
\text { Madohomé } \\
\text { Bessissan } \\
\text { Don tsi } \\
\text { Nugui } \\
\text { Gbéli } \\
\text { Sasaplala } \\
\text { Coclo ta dé } \\
\text { Baba ti djin } \\
\text { Agnagna } \\
\text { Adouwadzi } \\
\text { Sisinon } \\
\text { Tronsi poha } \\
\text { Fonyi tsi }\end{array}$ & $\begin{array}{c}G \\
F \\
F \\
F ; G ; P A \\
P E \\
F r \\
P E \\
F \\
F \\
P A \\
F r \\
P A \\
\text { R } \\
\text { T } \\
P E \\
F\end{array}$ & $\begin{array}{c}A L \\
A L \\
A L \\
A L ; \text { décoction } \\
\text { Décoction } \\
A L \\
\text { Décoction } \\
\text { Infusion } \\
\text { Décoction } \\
A L \\
A L \\
\text { Décoction } \\
A L \\
\text { Décoction } \\
\text { Infusion } \\
\text { Infusion }\end{array}$ \\
\hline 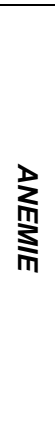 & $\begin{array}{l}\text { Adansonia digitata } \\
\text { Byrsocarpus coccineus } \\
\text { Hibiscus sabdariffa } \\
\text { Jatropha gossypifolia } \\
\text { Lannea kerstengii } \\
\text { Paullinia pinnata } \\
\text { Piliostigma thonningii } \\
\text { Pteleopsis suberosa } \\
\text { Sorghum bicolor } \\
\text { Uvaria chamae } \\
\text { Vernonia amygdalina } \\
\text { Vitellaria paradoxa } \\
\text { Waltheria Indica }\end{array}$ & $\begin{array}{c}\text { Bombacaceae } \\
\text { Connaraceae } \\
\text { Malvaceae } \\
\text { Euphorbiaceae } \\
\text { Anacardiaceae } \\
\text { Sapindaceae } \\
\text { Ceaesalpiniaceae } \\
\text { Combretaceae } \\
\text { Gramineae } \\
\text { Annonaceae } \\
\text { Compositeae } \\
\text { Sapotaceae } \\
\text { Sterculiaceae }\end{array}$ & $\begin{array}{l}\text { Adido tsi } \\
\text { Tomegavi } \\
\text { Gnanto dzen } \\
\text { Baba ti djin } \\
\text { Melonkou } \\
\text { Okuika } \\
\text { Eklon } \\
\text { Sisinon } \\
\text { Adako } \\
\text { Agba nan tsi } \\
\text { Aloma } \\
\text { Yokou tsi } \\
\text { Adouwè tsi }\end{array}$ & $\begin{array}{c}F ; T \\
R \\
P A \\
F \\
T \\
F+R \\
F \\
T \\
\text { Gaine } \\
E+F \\
P A \\
T \\
F+R\end{array}$ & $\begin{array}{c}\text { Infusion ; décoction } \\
\text { Infusion } \\
\text { Décoction sommaire } \\
\text { Infusion } \\
\text { Décoction } \\
\text { Décoction sommaire } \\
\text { Infusion } \\
\text { Décoction } \\
\text { Infusion } \\
\text { Décoction } \\
\text { Infusion } \\
\text { Décoction } \\
\text { Décoction }\end{array}$ \\
\hline
\end{tabular}




\begin{tabular}{|c|c|c|c|c|c|}
\hline ज్ & $\begin{array}{c}\text { Boerhavia diffusa } \\
\text { Byrsocarpus coccineus } \\
\text { Cassytha filiformis } \\
\text { Croton zambesicus } \\
\text { Holarrhena floribunda } \\
\text { Kigelia africana } \\
\text { Lonchocarpus cyanescens } \\
\text { Phyllanthus amarus } \\
\text { Phyllanthus niruri } \\
\text { Plumbago zeylanica } \\
\text { Pteleopsis suberosa } \\
\text { Spathodea campanulata }\end{array}$ & $\begin{array}{l}\text { Nyctaginaceae } \\
\text { Connaraceae } \\
\text { Lauraceae } \\
\text { Euphorbiaceae } \\
\text { Apocynaceae } \\
\text { Bignoniaceae } \\
\text { Papillioonaceae } \\
\text { Euphorbiaceae } \\
\text { Euphorbiaceae } \\
\text { Plumbaginaceae } \\
\text { Combretaceae } \\
\text { Bignoniaceae }\end{array}$ & $\begin{array}{l}\text { Ahozéméklo } \\
\text { Tomegavi } \\
\text { Teklimadzoeka } \\
\text { Gbéli } \\
\text { Sé Sé hou } \\
\text { Gna kpé kpé } \\
\text { Aho tsi } \\
\text { Ehlin } \\
\text { Hlenwe } \\
\text { Adouwadzi } \\
\text { Sisinon } \\
\text { Adassi golo }\end{array}$ & $\begin{array}{c}\text { PA } \\
R \\
P E \\
R \\
T \\
R \\
R \\
P A \\
P A \\
R \\
T \\
T\end{array}$ & $\begin{array}{l}\text { Décoction } \\
\text { Décoction } \\
\text { Infusion } \\
\text { Décoction } \\
\text { Décoction } \\
\text { Décoction } \\
\text { Décoction } \\
\text { Infusion } \\
\text { Infusion } \\
\text { Alcoolature } \\
\text { Décoction } \\
\text { Décoction }\end{array}$ \\
\hline 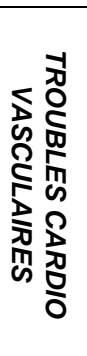 & $\begin{array}{c}\text { Aerva lanata } \\
\text { Anthocleista djalonnensis } \\
\text { Byrsocarpus coccineus } \\
\text { Cardiospermum halicacabum } \\
\text { Clausena anisata } \\
\text { Commiphora africana } \\
\text { Cryptolepis sanguinolenta } \\
\text { Gardenia ternifolia } \\
\text { Heliotropium indicum } \\
\text { Lactuca taraxacifolia. } \\
\text { Lippia multiflora }\end{array}$ & $\begin{array}{l}\text { Amaranthaceae } \\
\text { Loganiaceae } \\
\text { Connaraceae } \\
\text { Sapindaceae } \\
\text { Rutaceae } \\
\text { Burseraceae } \\
\text { Periplocaceae } \\
\text { Rubiaceae } \\
\text { Borraginaceae } \\
\text { Compositeae } \\
\text { Verbaceae }\end{array}$ & $\begin{array}{l}\text { Akoèma } \\
\text { Gbolo ba } \\
\text { Tomegavi } \\
\text { Otoka } \\
\text { Ida } \\
\text { Nugui } \\
\text { Eke dzen } \\
\text { Fliflé tsi } \\
\text { Coclo ta dé } \\
\text { Awonto } \\
\text { Avloti }\end{array}$ & $\begin{array}{l}\mathrm{PE} \\
T+\mathrm{R} \\
\mathrm{PA} \\
\text { Lianes } \\
\mathrm{R} \\
\text { Tiges } \\
\mathrm{R} \\
\mathrm{F} \\
\mathrm{PA} \\
\mathrm{F} \\
\mathrm{PA} \\
\end{array}$ & $\begin{array}{c}\text { Infusion } \\
\text { Décoction } \\
\text { Infusion } \\
\text { Infusion } \\
\text { Décoction } \\
\text { Infusion } \\
\text { Décoction sommaire } \\
\text { Infusion } \\
\text { Infusion } \\
\text { Infusion } \\
\text { Infusion } \\
\end{array}$ \\
\hline
\end{tabular}




\section{Modes d'utilisation et parties utilisées}

Parmi les différentes parties de plantes utilisées pour obtenir les préparations servant dans le traitement des différentes pathologies, les feuilles viennent en tête $(40,5 \%)$ suivies des racines $(35,8 \%)$, de la partie aérienne $(32,07 \%)$, et de la plante entière $(21,6 \%)$ (Figure 3$)$.

Toutes ces différentes parties sont utilisées dans divers modes de préparation. Le mode de préparation le plus fréquent est l'infusion, suivi de la décoction, de la macération, de l'application locale, de l'alcoolature et de l'ingestion. La calcination, la poudre, la fumigation et le pillât à priser sont très faiblement utilisés (Figure 4).

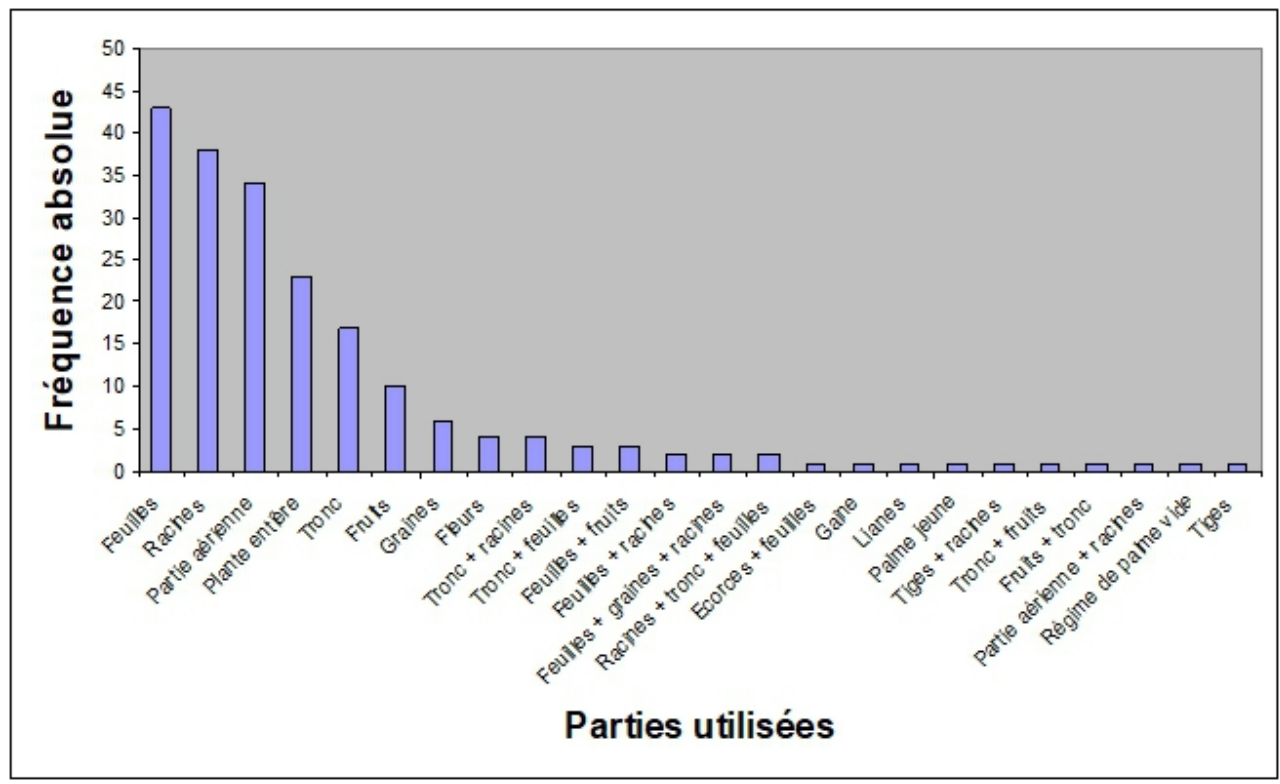

Figure 3. Fréquence d'utilisation des différentes parties des plantes

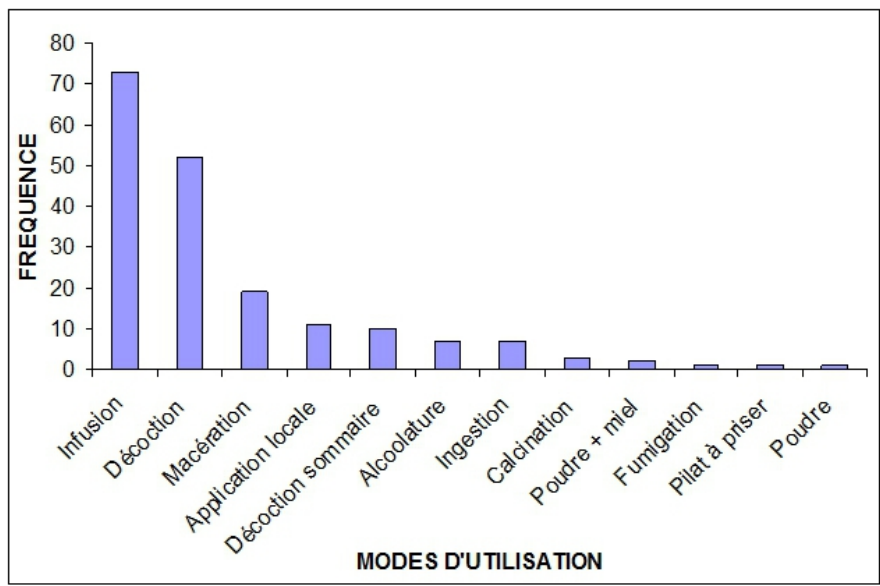

Figure 4. Les fréquences des différents modes d'utilisation des plantes. 


\section{Discussion}

Cent-six (106) espèces ont été recensées sur les marchés visités. Ce nombre, quoique inférieur à celui de Tossou (1998) qui a recensé deux cent seize (216) espèces, est assez significatif. En effet, alors que l'étude de Tossou (1998) est menée sur vingt-six (26) marchés de la ville de Lomé, seulement neuf (9) marchés ont été visités pour la présente étude. En outre elle enrichi la liste des plantes médicinales répertoriées par Tossou (1998) de vingt-et-une (21) nouvelles espèces dont les plus marquantes sont: Aerva lanata, Antiaris africana, Argemone mexicana, Borassus aethiopum, Chassalia kolly.

Parmi les familles les plus représentées, on a les Caesalpiniaceae et les Euphorbiaceae, résultats qui s'accordent sur le plan de la botanique systématique avec ceux obtenus sur les flores tropicales notamment par Hutchinson et Dalziel (1954-1972), Brunel et al. (1984), Walters et Hamilton (1994). La grande majorité des espèces recensées dans cette étude est retrouvée dans l'ouvrage de Iwu (1993).

Les indications thérapeutiques citées dans cette étude sont très nombreuses comparées aux résultats obtenus par Tossou (1998). En effet, elle dénombre vingt-et-une (21) pathologies, tandis que dans cette étude on en compte soixante-trois (nombre obtenu après regroupement des nombreuses pathologies citées sur le terrain). Les feuilles constituent la partie de la plante la plus utilisée. La même observation a été faite dans les études d'Assouma et al. (2018).

Ces résultats s'accordent aussi avec les Autorités de Santé qui parlent d'un engouement des populations pour la médecine traditionnelle et, ceci, pas uniquement au Togo.

Dans cette étude-ci, comme dans celle de Tossou (1998), le paludisme est l'une des pathologies le plus rencontrée sur le terrain. Cette pathologie est, selon l'OMS, la première cause de mortalité en Afrique. Aujourd'hui, les remèdes les plus efficaces contre le paludisme en pharmacie sont vendus entre 2.500 FCFA et 5.000 FCFA; ce qui les rend inaccessibles pour une grande majorité de Togolais. Cela explique l'importance de cette pathologie en phytothérapie.

En outre, en étudiant les tranches de la population qui se traitent auprès des tradithérapeutes, on se rend compte que les personnes les moins rencontrées ont entre 35 et 55 ans. Ce sont des personnes encore en activité, avec une couverture sociale leur permettant d'acheter des médicaments en pharmacie. On peut donc penser que le regain que connaît la médecine traditionnelle aujourd'hui est dû à la crise économique actuelle.

Nous nous sommes heurtées à un manque de coopération des clients. Il aurait peut-être fallu remettre les fiches de renseignements aux tradithérapeutes, qui auraient courtoisement demandé à leurs patients de les remplir. 
Certains des résultats obtenus sont confirmés et complétés par d'autres travaux antérieurs. C'est ainsi que Carpentier (2004), confirme que Paullinia pinnata est utilisée dans les cas d'asthénie sexuelle et même d'impuissance masculine et, qu'en plus de l'anémie, cette plante traite également les maladies sexuellement transmissibles, les hémorragies, la dysenterie.

L'utilisation d'Annona senegalensis est confirmée comme plante antitussive, antidiarrhéique par Bandiaré (1998) qui ajoute que la même plante est utilisée dans le traitement des maladies du SNS comme Maguifera indica, Carica papaya et Euphorbia hirta.

À propos du traitement de la constipation par Vernonia amygdalina, les résultats obtenus s'accordent avec ceux de Laurian (1994), quant à Pteleopis suberosa, les travaux de Batawila (2002), confirment son usage traditionnel dans le traitement des dermatoses et des ulcères gastro-duodénaux. Et d'après Baba-Moussa (1999), son usage dans le traitement des dermatoses serait dû à son activité antifongique puissante.

Il faut également préciser que la plupart de ces plantes recensées font l'objet de plusieurs études antérieures sur le plan chimique et pharmacologique en vue de justifier leurs utilisations traditionnelles.

\section{Conclusion}

L'enquête ethnobotanique menée sur neuf marchés de la région maritime a permis de recenser 106 espèces médicinales regroupées en 61 familles et 97 genres dont 90 dicotylédones et 6 monocotylédones et une fougère.

Ces différentes espèces interviennent dans le traitement de soixantetrois pathologies dont le paludisme et les troubles gynécologiques sont les plus fréquentes. Cette grande panoplie d'indications thérapeutiques montre le rôle important que jouent les plantes sur le plan médical.

L'infusion et la décoction apparaissent comme les modes de préparation les plus utilisés par les tradithérapeutes. Plusieurs parties de plantes entrent dans ces différentes préparations, les feuilles étant les parties les plus utilisées.

Malgré la volonté des autorités africaines à asseoir une politique sectorielle de santé pour satisfaire les populations, les pratiques ancestrales demeurent dans un contexte de pluralité et de diversité. Il s'avère donc nécessaire aujourd'hui de disposer d'une pharmacopée propre à l'Afrique. Cette étude constitue une piste de recherche dans la mesure où elle permet une orientation dans le choix des plantes cibles pour des pathologies données.

\section{References:}

1. Assouma, A. F., Koudouvo, K., Diatta, W., Vidzro, M.K., Guelly, A.K., Dougnon, J., Agbonon, A., Tozo, K., \& Gbeassor, M. (2018). 
Enquête Ethnobotanique sur la Prise en charge traditionnelle de l'infertilité féminine dans la région sanitaire des savanes au Togo. European Scientific Journal January edition Vol.14, No.3 ISSN: 1857 - 7881 (Print) e - ISSN 1857- 7431

2. Baba-Moussa, F.A.K. (1999). Recherches sur les propriétés antifongiques de plantes utilisées en médicine traditionnelle au Bénin et au Togo - Thèse Doctorat - Université de Reims - 186 pages.

3. Bandiaré, A.F. (1998). Etude bibliographique de quelques planes du Niger utilisées en Médecine traditionnelle, réputées actives ou potentiellement actives sur certaines maladies du système nerveux central - Thèse Doctorat - Université de Reims - 92 pages.

4. Batawila, K. (2002). Diversité, écologie et propriétés antifongiques des combretacées du Togo - Thèse Doctorat - Université de Reims et de Lomé - 143 pages

5. Batawila, K. (2005). Etude ethnobotanique sur les plantes légumières de cueillette au Togo ; Mémoire DUEC Ethnobotanique appliquée Université de Lille - 53 pages.

6. Brunel, J.F., Hiekpo, P., \& Scholz, H. (1984). Flore analytique du Togo : Phanérogames, - Edition Eschborn - 751 pages.

7. Carpentier, M. (2004). Plantes et dysfonction érectile: Etude phytochimique et biologique de Paullinia pinnata L. (Sapindacées) Université de Lille - 71 pages.

8. Encyclopédie WIKIPEDIA : http://fr.wikipedia.org/wiki/Togo Consulté le 06-06-07

9. Hutchinson, J. \& Dalziel, J.M. (1954 - 1972). Flora of West Tropical Africa $-2^{\text {nd }}$ Ed. Revised by Keay and Hepper, 3 vol.

10. Iwu, M.M. (1993). Handbook of African Medicinal Plants - CRS Press. Boca Raton - 435 pages.

11. Laurian, G. (1994). Etudes ethnobotaniques et ethnopharmacologiques des plantes du Bénin et du Togo - Thèse Doctorat - Université de Reims -76 pages.

12. Sari (2006). Contribution à l'études des propriétés antifongiques de Elaeophorbia grandifolia, Ficus polita, Microgyna inernis : trois espèces végétales du Togo - Thèse Doctorat - Université de Lomé Togo - 55 pages.

13. Tossou, M.G. (1998). Quelques espèces botaniques du prélèvement et la commercialisation des plantes médicinales dans la ville de Lomé (Togo) - Mémoire de DEA Biologie végétale appliquée - Université du Bénin,Togo - 68 pages.

14. Walters, M. \& Hamilton, A. (1994). La diversité végétale : une source de richesse vitale - WWF Ed. - 47 pages. 